\title{
Order Relation on the Permutation Symbols in the Ehresmann Subvariety Class Associated to the Distinguished Monomials of Flag Manifolds
}

\author{
Praise Adeyemo, Samuel Ilori \\ Department of Mathematics, University of Ibadan, Ibadan, Nigeria \\ Email: ph.adeyemo@mail.ui.edu.ng, ailori1@yahoo.com
}

Received June 2, 2013; revised July 8, 2013; accepted August 5, 2013

Copyright (C 2013 Praise Adeyemo, Samuel Ilori. This is an open access article distributed under the Creative Commons Attribution License, which permits unrestricted use, distribution, and reproduction in any medium, provided the original work is properly cited.

\begin{abstract}
In this paper, we use the theory of lexicographical and graded lexicographical orders to compare two distinguished monomials through their codes of invariants $\mathbb{Z}_{\geq 0}^{n}$ and study the effect of this comparison on their respective defining permutation symbols in the Ehresmann subvariety classes.
\end{abstract}

Keywords: Ehresmann Subvariety; Borel-Hirzebruch Basis; Flag Manifold; Intersection Formula

\section{Introduction}

A flag $F$ is a nested system

$$
F: F_{0} \subset F_{1} \subset F_{2} \subset \cdots \subset F_{n-1} \subset F_{n}
$$

$\operatorname{dim} F_{i}=i, \quad 0 \leq i \leq n$ of subspaces of $P(V)$, the projective space of an $(n+1)$-dimensional vector space $V$ over $\mathbb{C}$, the field of complex numbers. The set of all such flags is called flag manifold and will be denoted by $F(n+1)$. The general linear group $G L(n+1, \mathbb{C})$ acts transitively on $F(n+1)$. Let $E$ be a fixed reference flag in $F(n+1)$. The isotropic group of $E$ is a Borel subgroup $B$ so that

$$
F(n+1) \cong G L(n+1, \mathbb{C}) / B .
$$

Its dimension is $\frac{n(n+1)}{2}$. The flag manifold $F(n+1)$ is the disjoint union of $B$-orbits indexed by elements of symmetric group $S_{n}$

$$
F(n+1) \cong G L(n+1, \mathbb{C}) / B=\bigsqcup_{w \in S_{n}} B \cdot w B .
$$

The major interest in this direction has been on the cohomology of these manifolds, where by cohomology, we mean in a general sense; singular and equivariant, K-theory and equivariant K-theory. For each of these theories, there are two descriptions of cohomology. One is in terms of Ehresmann classes, which are cohomology associated to the Ehresmann subvarieties of $F(n+1)$ given in terms of permutation symbols. There is one Ehresmann class for each permutation symbol [1]. The Ehresmann classes form a basis for the cohomology over its ground ring and the other is in terms of generators and relations called the Borel-Hirzebruch basis elements [2].

Definition 1. Let

$$
S: S_{0} \subset S_{1} \subset \cdots \subset S_{n}, \quad \operatorname{dim} S_{j}=j
$$

be a fixed flag. An Ehresmann symbol is a matrix

$$
\left[\begin{array}{cccc}
a_{00} & & & \\
a_{10} & a_{11} & & \\
\vdots & & \ddots & \\
a_{n-1,0} & a_{n-1,1} & \cdots & a_{n-1, n-1}
\end{array}\right]
$$

where $a_{i j}$ are the integers such that

$$
0<a_{i 0}<a_{i 1}<\cdots<a_{i i} \leq n \quad(i=0, \cdots, n) .
$$

Following Monk [3], the $i^{\text {th }}$ row of this symbol is to be interpreted as a Schubert condition $\left[a_{i 0}, a_{i 1}, \cdots, a_{i i}\right]$ on the element $F_{i}$ of $F$. The matrix represents a subvariety of $F(n+1)$ consisting of all the flags $F$ satisfying the conditions:

$$
\operatorname{dim}\left(F_{i} \cap S_{a_{i j}}\right) \geq j \quad(0 \leq j \leq i \leq n)
$$

Definition 2. The variety of $F(n+1)$ is said to be irreducible(and the corresponding symbol is called an 
irreducible symbol) if for every $a_{i j}(j<i<n-1)$, there exists $k \geq j$ such that $a_{i j}=a_{i+1}, k$.

The set of all such irreducible varieties is called the Ehresmann base.

Remark 1. Writing a matrix for each irreducible symbol is unwieldy and Monk [3] suggested representing the matrix by a permutation $\left(a_{0}, \cdots, a_{n}\right)$ of $0,1, \cdots, n$ where $a_{i}$ is the new element in the $i^{\text {th }}$ row and $a_{n}$ is the missing integer. Conversely every permutation of $0,1, \cdots, n$ determines an irreducible symbol and hence the number of elements in the Ehresmann base is $(n+1)$ !.

It has been proved that the dimension of the subvariety represented by the matrix when irreducible is

$$
\begin{aligned}
& \sum_{i=0}^{n-1}\left(a_{i}-m_{i}\right), \\
& m_{i}=\#\left\{a_{j}: a_{j}<a_{i}, 0 \leq j<i \leq n-1\right\}
\end{aligned}
$$

\section{Distinguished Monomials}

It is well known in [4-6] that the flag manifold $F(n+1)$ comes equipped with a flag of tautological vector bundles $E_{0} \subset E_{1} \subset \cdots \subset E_{n}$ and associated sequence of line bundles $L_{i}=E_{i+1} / E_{i}, i=0, \cdots, n$. The $L_{i}$ possess natural hermitian structures induced from the standard hermitian metric $\sum z_{i} \bar{z}_{i}$ on $(n+1)$-dimensional vector space $V$ over $\mathbb{C}$. For $i=0, \cdots, n$, we denote by $\gamma_{i}$, the 2 -dimensional Chern form on $F(n+1)$ of the hermitian line bundle $L_{i}$ [7-9]. In other words, they represent the Chern classes $c_{1}\left(L_{i}\right)$ in the cohomology of $F(n+1)$. The only nontrivial Chern class is the first Chern class, which is an element of the second cohomology group of the manifold [10]. The cohomology ring $H^{*}(F(n+1), \mathbb{Z})$ is therefore, generated by the Chern classes $c_{1}\left(L_{i}\right)=\gamma_{i}$.

There is indeed a correspondence between the permutation symbols and the $\gamma^{\prime} s$, viz,

$$
\left(a_{0}, a_{1}, \cdots, a_{n}\right) \leftrightarrow\left(\gamma_{0}, \gamma_{1}, \cdots, \gamma_{n}\right)
$$

and it is interesting to note that any permutation symbol can be identified uniquely with certain product of these generators. These specialized products are called the distinguished monomials.

Definition 3. Let $T_{d}=\left(a_{0}, \cdots, a_{n}\right)$ be any cycle of the Ehresmann subvariety class of dimension $d$ in the cohomology of the flag manifold $F(n+1)$, then the product $\prod_{i=0}^{n-1} \gamma_{i}^{\beta_{i}}$ is the distinguished monomial of $T_{d}$ where $\beta_{i}=\chi\left(a_{i}\right)$, that is,

$$
\beta_{i}=\#\left\{a_{k}: a_{k}>a_{i}, i<k \leq n\right\}
$$

Example 1. The distinguished monomial of the cycle
$N_{2}=(1203)$ in the Ehresmann cycle class of dimension 2 of the cohomology of $\mathbf{F ( 4 )}$ is given by $\gamma_{0}^{2} \gamma_{1} \gamma_{2}$.

Definition 4. The degree $\beta$ of the distinguished monomial $\prod_{i=0}^{n-1} \gamma_{i}^{\beta_{i}}$ is given by $\beta=\sum_{i=0}^{n-1} \chi\left(a_{i}\right)$, the index of the cycle $\left(a_{0}, \cdots, a_{n}\right)$, that is, $\beta=\sum_{i=0}^{n-1} \beta_{i}$.

The collection of distinguished monomials is denoted by $\mathcal{M}_{n+1}$

\section{Main Results}

We now compare any two distinguished monomials and study the effect of this comparison on their respective defining cycles via the code of invariants $\mathbb{Z}_{\geq 0}^{n}$, the collection of $n$-tuple exponents of distinguished monomials. In order to this, we impose ordering on these monomials. In practice, we shall assume the following relation on the generators $\gamma_{0}, \gamma_{1}, \cdots, \gamma_{n-1}$

$$
\gamma_{0}>\gamma_{1}>\cdots>\gamma_{n-1}
$$

Several orderings can be defined on set of monomials but due to the characterization of $\mathcal{M}_{n+1}$, it seems lexicographic order and graded lexicographic order are most appropriate.

Definition 5 (Lexicographic Order). Let

$$
\alpha=\left(\alpha_{1}, \alpha_{2}, \cdots, \alpha_{n}\right) \text { and } \beta=\left(\beta_{1}, \beta_{2}, \cdots, \beta_{n}\right) \in \mathbb{Z}_{\geq 0}^{n},
$$

the collection of $n$-tuple exponents of distinguished monomials. $\alpha>_{\text {lex }} \beta$ if in the vector difference $\alpha-\beta \in \mathbb{Z}^{n}$, the left-most nonzero entry is positive. We shall write

$$
\prod_{i=0}^{n-1} \gamma_{i}^{\alpha_{i}}>_{l e x} \prod_{i=0}^{n-1} \gamma_{i}^{\beta_{i}}
$$

if $\alpha>_{\text {lex }} \beta$

$$
\forall \prod_{i=0}^{n-1} \gamma_{i}^{\alpha_{i}}, \prod_{i=0}^{n-1} \gamma_{i}^{\beta_{i}} \in \mathcal{M}_{n+1}
$$

Definition 6 (Graded lexicographic Order). Let $\alpha, \beta \in \mathbb{Z}_{\geq 0}^{n}$, the collection of $n$-tuple exponents of distinguished monomials. We say

$$
\prod_{i=0}^{n-1} \gamma_{i}^{\alpha_{i}}>_{\text {grlex }} \prod_{i=0}^{n-1} \gamma_{i}^{\beta_{i}}
$$

if

$$
|\alpha|=\sum_{i=0}^{n-1} \alpha_{i}>|\beta|=\sum_{i=0}^{n-1} \beta_{i},
$$

or $|\alpha|=|\beta|$ and $\alpha>_{\text {lex }} \beta$.

The distinguished monomial ordering relation on $>$ on the code of invariants $\mathbb{Z}_{\geq 0}^{n}$, the set of $n$-tuple of collection of monomials is well-ordered. By the distinguished monomial ordering relation on $\mathbb{Z}_{\geq 0}^{n}$ in this context, we mean graded lexicographic order on $\mathbb{Z}_{\geq 0}^{n}$ and denote it by $>$.

Definition 7. Let $\left(a_{0}, \cdots, a_{n}\right)$ and $\left(b_{0}, \cdots, b_{n}\right)$ be 
any two cycles in the Ehresmann cycle class

$$
\left[\left(a_{0}, \cdots, a_{n}\right), F(n+1)\right]_{d}
$$

of dimension $d$. We say

$$
\left(a_{0}, \cdots, a_{n}\right)>\left(b_{0}, \cdots, b_{n}\right)
$$

if

$$
\left(a_{0}, \cdots, a_{n}\right)>_{l e x}\left(b_{0}, \cdots, b_{n}\right) .
$$

Remark 2. In general, the ordering extends over the the Ehresmann base $\mathcal{E}_{n+1}$. In other words, the ordering still holds even if the cycles are not equivalent.

Lemma 1. If $\left(a_{0}, a_{1}, \cdots, a_{n}\right)$ and $\left(b_{0}, b_{1}, \cdots, b_{n}\right)$ are any two irreducible symbols of the Ehresmann subvarieties in the the flag manifold $F(n+1)$, then $\left(a_{0}, a_{1}, \cdots, a_{n}\right)$ is equivalent to $\left(b_{0}, b_{1}, \cdots, b_{n}\right)$ if and only if

$$
\sum_{i=0}^{n-1}\left(a_{i}-m_{i}\right)=\sum_{i=0}^{n-1}\left(b_{i}-m_{i}\right)
$$

where

$$
\begin{aligned}
m_{i} & =\#\left\{a_{k}\left(\text { resp. } b_{k}\right): a_{k}\left(\text { resp. } b_{k}\right)\right. \\
& \left.<a_{i}\left(\text { resp. } b_{i}\right), 0 \leq k \leq i-1\right\} .
\end{aligned}
$$

Remark 3. Equivalence of permutation symbols is an equivalence relation. Each of the partitions is called the Ehresmann cycle class and denoted by

$$
\left[\left(a_{0}, \cdots, a_{n}\right) ; F\right]_{d}
$$

where $d$ is the dimension of the class and hence the flag manifold $F(n+1)$ is given by the disjoint union:

$$
F(n+1)=\coprod_{d=0}^{\operatorname{dim} F(n+1)}\left[\left(a_{0}, \cdots, a_{n}\right) ; F\right]_{d}
$$

Theorem 1. Let $\left[\left(a_{0}, \cdots, a_{n}\right) ; F(n+1)\right]_{d}$ be an Ehresmann cycle class of the flag manifold ${ }^{d} F(n+1)$. Let $\mathfrak{T}_{n+1}$ be the subcollection of the distinguished monomials of degree $\beta$ of $\mathcal{H}_{n+1}$ in the cohomology ring of the manifold $F(n+1)$. Then the dimension of of the class $\left[\left(a_{0}, \cdots, a_{n}\right) ; F\right]$ is expressed in terms of the degree of the monomials, that is

$$
\operatorname{dim}\left[\left(a_{0}, \cdots, a_{n}\right) ; F\right]=\frac{n(n+1)}{2}-\sum_{i=0}^{n-1} \chi\left(a_{i}\right) .
$$

Proof. The dimension of any Ehresmann cycle class in the flag manifold $F(n+1)$ has been proved by Ehresmann[3] and given by

$$
\operatorname{dim}\left[\left(a_{0}, \cdots, a_{n}\right) ; F\right]=\sum_{i=0}^{n+1}\left(a_{i}-m_{i}\right)
$$

where $m_{i}=\#\left\{a_{j}: a_{j}<a_{i}, 0 \leq j<i \leq n-1\right\}$. Extending the summation to accommodate $i=n$ automatically puts $m_{n}=a_{n}$ which makes equation 8 still stable. In this case, $\sum_{i=0}^{n} m_{i}$ turns out to be index ind $\left(a_{0}, \cdots, a_{n}\right)$ of any cycle $\left(a_{0}, \cdots, a_{n}\right)$ in the class $\left[\left(a_{0}, \cdots, a_{n}\right) ; F\right]$ given by $\sum_{i=0}^{n-1} \chi\left(a_{i}\right)$ which coincides with the degree of the distinguished monomial of the cycle. The $\sum_{i=0}^{n} a_{i}$ is precisely the dimension of the flag manifold $F(n+1)$, that is, $\frac{n(n+1)}{2}$ and hence

$$
\operatorname{dim}\left[\left(a_{0}, \cdots, a_{n}\right) ; F\right]=\frac{n(n+1)}{2}-\sum_{i=0}^{n-1} \chi\left(a_{i}\right) .
$$

Theorem 2. Let

$$
\left[\left(a_{0}, \cdots, a_{n}\right) ; F(n+1)\right]_{d}
$$

be the Ehresmann cycle class of dimension $d$ in the cohomology of $F(n+1)$, and let

$$
\mathcal{E}_{n+1}=\coprod_{d=0}^{\operatorname{dim} F(n+1)}\left[\left(a_{0}, \cdots, a_{n}\right) ; F(n+1)\right]_{d}
$$

be the disjoint union of such classes. Let

$$
\mathcal{M}_{n+1}=\coprod_{\beta=0}^{\operatorname{dim} F(n+1)} \mathfrak{H}_{\beta}
$$

be the graded monoid of distinguished monomials of degrees $\beta$ in the cohomology ring of the flag manifold $F(n+1)$. Then there is a natural bijection

$$
\phi:\left[\left(a_{0}, \cdots, a_{n}\right) ; F\right]_{d} \mapsto \mathfrak{H}_{\beta}
$$

between $\mathcal{E}_{n+1}$ and $\mathcal{M}_{n+1}$.

Proof

We define a map

$$
\phi: \mathcal{E}_{n+1} \rightarrow \mathcal{M}_{n+1}
$$

by

$$
\phi\left(\left[\left(a_{0}, \cdots, a_{n}\right) ; F(n+1)\right]_{d}\right)=\mathfrak{H}_{\beta}
$$

where $\mathfrak{H}_{\beta}$ is a subcollection of $\mathcal{M}_{n+1}$, that is,

$$
\mathfrak{H}_{\beta}=\left\{\prod_{i=0}^{n-1} \gamma_{i}^{\beta_{i}} \in \mathcal{M}_{n+1}: \operatorname{deg}\left(\prod_{i=0}^{n-1} \gamma_{i}^{\beta_{i}}\right)=\beta, 0 \leq i \leq n-1\right\} .
$$

Let

$$
\phi\left(\left[\left(a_{0}, \cdots, a_{n}\right) ; F(n+1)\right]_{k}\right)=\mathfrak{H}_{\beta}
$$

and

$$
\begin{aligned}
& \phi\left(\left[\left(b_{0}, \cdots, b_{n}\right) ; F(n+1)\right]_{k^{\prime}}\right)=\mathfrak{H}_{\beta^{\prime}}^{\prime}, \\
& 0 \leq \beta, \beta^{\prime} \leq \frac{n(n+1)}{2},
\end{aligned}
$$




$$
0 \leq k, k^{\prime} \leq \operatorname{dim}(F(n+1)) .
$$

Suppose that

$$
\left[\left(a_{0}, \cdots, a_{n}\right) ; F(n+1)\right]_{k}=\left[\left(b_{0}, \cdots, b_{n}\right) ; F(n+1)\right]_{k^{\prime}}
$$

which implies that

$$
\sum_{i=0}^{n+1}\left(a_{i}-m_{i}\right)=\sum_{i=0}^{n+1}\left(b_{i}-t_{i}\right)
$$

where

$$
\begin{aligned}
& m_{i}=\#\left\{a_{j}: a_{j}<a_{i}, 0 \leq j<i \leq n-1\right\} \\
& t_{i}=\#\left\{b_{j}: b_{j}<b_{i}, 0 \leq j<i \leq n-1\right\}
\end{aligned}
$$

From the Theorem 1,

$$
\frac{n(n+1)}{2}-\sum_{i=0}^{n-1} \chi\left(a_{i}\right)=\frac{n(n+1)}{2}-\sum_{i=0}^{n-1} \chi\left(b_{i}\right)
$$

and hence

$$
\sum_{i=0}^{n-1} \chi\left(a_{i}\right)=\sum_{i=0}^{n-1} \chi\left(b_{i}\right)
$$

which implies that

$$
\begin{aligned}
& \phi\left(\left[\left(a_{0}, \cdots, a_{n}\right) ; F(n+1)\right]_{k}\right) \\
& =\phi\left(\left[\left(b_{0}, \cdots, b_{n}\right) ; F(n+1)\right]_{k^{\prime}}\right)
\end{aligned}
$$

Therefore, $\phi$ is well defined.

Suppose that

$$
\begin{aligned}
& \phi\left(\left[\left(a_{0}, \cdots, a_{n}\right) ; F(n+1)\right]_{k}\right) \\
& =\phi\left(\left[\left(b_{0}, \cdots, b_{n}\right) ; F(n+1)\right]_{k^{\prime}}\right)
\end{aligned}
$$

in other words $\mathfrak{H}_{\beta}=\mathfrak{H}_{\beta^{\prime}}^{\prime}$

$$
\begin{aligned}
& \Rightarrow \sum_{i=0}^{n-1} \chi\left(a_{i}\right)=\sum_{i=0}^{n-1} \chi\left(b_{i}\right) \\
& \Rightarrow \frac{n(n+1)}{2}-\sum_{i=0}^{n-1} \chi\left(a_{i}\right)=\frac{n(n+1)}{2}-\sum_{i=0}^{n-1} \chi\left(b_{i}\right) \\
& \Rightarrow k=k^{\prime}
\end{aligned}
$$

and therefore,

$$
\begin{aligned}
& {\left[\left(a_{0}, \cdots, a_{n}\right) ; F(n+1)\right]_{k}} \\
& =\left[\left(b_{0}, \cdots, b_{n}\right) ; F(n+1)\right]_{k^{\prime}}
\end{aligned}
$$

and hence $\phi$ is injective.

For any subcollection $\mathfrak{H}_{\beta}$ in $\mathcal{M}_{n+1}$. By definition, $\beta=\sum_{i=0}^{n-1} \chi\left(a_{i}\right)$ implies that $\frac{n(n+1)}{2}-\sum_{i=0}^{n-1} \chi\left(a_{i}\right)$ is the dimesion of the Ehresmann class

$$
\left[\left(a_{0}, \cdots, a_{n}\right) ; F(n+1)\right] \text { in } \coprod_{d=0}^{\operatorname{dim} F(n+1)}\left[\left(a_{0}, \cdots, a_{n}\right)\right]
$$

such that

$$
\phi\left(\left[\left(a_{0}, \cdots, a_{n}\right) ; F(n+1)\right]\right)=\mathfrak{H}_{\beta} .
$$

Theorem 3. If the distinguished monomials of two cycles $\mathfrak{N}=\left(a_{0}, \cdots, a_{n}\right)$ and $\mathfrak{A}=\left(b_{0}, b_{1}, \cdots, b_{n}\right)$ in the the Ehresmann base $\mathcal{E}_{n+1}$ are equal then the two cycles coincide.

\section{Proof}

In other words, the theorem says no two distinct cycles share the same distinguished monomial. Suppose that $\left(a_{0}, a_{1}, \cdots, a_{n}\right)$ and $\left(b_{0}, \cdots, b_{n}\right)$ are not equivalent in the sense of Lemma 2, this leads to the fact that

$$
\sum_{i=0}^{n-1} \chi\left(a_{i}\right) \neq \sum_{i=0}^{n-1} \chi\left(b_{i}\right)
$$

and hence different distinguished monomials. Now suppose they are equivalent, this implies that

$$
\sum_{i=0}^{n-1} \chi\left(a_{i}\right)=\sum_{i=0}^{n-1} \chi\left(b_{i}\right)=c .
$$

Consider the set $\mathcal{T}_{c}$ consisting of

$$
\left(\chi\left(a_{0}\right), \cdots, \chi\left(a_{n-1}\right)\right) \text { and }\left(\chi\left(b_{0}\right), \cdots, \chi\left(b_{n-1}\right)\right) \text {. }
$$

$\mathcal{T}_{c}$ is a subcollection of $\mathbb{Z}_{\geq 0}^{n}$ being the set of $n$-tuple exponents of distinguished monomials. Since $\mathbb{Z}_{\geq 0}^{n}$ is well ordered, $\mathcal{T}_{c}$ has a least element and therefore, the distinguished monomials defined by the two $n$-tuple exponents differ.

Corollary 1. If $\mathfrak{N}_{d}=\left(a_{0}, \cdots, a_{n}\right)$ is a cycle in the Ehresmann cycle class

$$
\left[\left(a_{0}, \cdots, a_{n}\right) ; F(n+1)\right]
$$

of dimension $d$. Then $\mathfrak{N}_{d}$ has at most one distinguished monomial.

Proof

Suppose $\mathfrak{N}_{d}=\left(a_{0}, \cdots, a_{n}\right)$ is identified with

$$
\prod_{i=0}^{n-1} \gamma_{i}^{\chi\left(a_{i}\right)} \text { and } \prod_{i=0}^{n-1} \gamma_{i}^{\chi\left(a_{i}^{\prime}\right)} \text {, }
$$

then the

$$
\operatorname{Ind}\left(a_{0}, \cdots, a_{n}\right)=\sum_{i=0}^{n-1} \chi\left(a_{i}\right)
$$

and

$$
\operatorname{Ind}\left(a_{0}, \cdots, a_{n}\right)=\sum_{i=0}^{n-1} \chi\left(a_{i}^{\prime}\right) .
$$

By the definition of $\operatorname{Ind}\left(a_{0}, \cdots, a_{n}\right)$, the subset $\mathfrak{T}_{c}$ consisting of

$$
\left(\chi\left(a_{0}\right), \cdots, \chi\left(a_{n-1}\right)\right) \text { and }\left(\chi\left(a_{0}^{\prime}\right), \cdots, \chi\left(a_{n-1}^{\prime}\right)\right)
$$

is singleton in $\mathbb{Z}_{\geq 0}^{n-1}$ and hence $\prod_{i=0}^{n-1} \gamma_{i}^{\chi\left(a_{i}\right)}$ and $\prod_{i=0}^{n-1} \gamma_{i}^{\chi\left(a_{i}^{\prime}\right)}$ coincide.

Using the definitions 5 and 6, we shall define ordering on the cycles of the Eheresmaan cycle class

$$
\left[\left(a_{0}, \cdots, a_{n}\right) ; F(n+1)\right]_{d}
$$


of dimension $d$ and give some of its intrinsic properties in relation to the corresponding subcollection $\mathcal{T}_{c}$ of distinguished monomials of degree $c$, where $c$ is given by

$$
c=\frac{1}{2}[n(n+1)-2 d]
$$

Definition 8. Let $\left(a_{0}, \cdots, a_{n}\right)$ and $\left(b_{0}, \cdots, b_{n}\right)$ be any two cycles in the Ehresmann cycle class

$$
\left[\left(a_{0}, \cdots, a_{n}\right) ; F(n+1)\right]_{d}
$$

of dimension $d$. We say

$$
\left(a_{0}, \cdots, a_{n}\right)>\left(b_{0}, \cdots, b_{n}\right)
$$

if

$$
\left(a_{0}, \cdots, a_{n}\right)>_{\text {lex }}\left(b_{0}, \cdots, b_{n}\right) .
$$

Remark 4. In general, the ordering extends over the Ehresmann base $\mathcal{E}_{n+1}$. In other words, the ordering still holds even if the cycles are not equivalent.

Definition 9. Let

$$
\left[\left(a_{0}, \cdots, a_{n}\right), F\right]_{d} \text { and }\left[\left(b_{0}, \cdots, b_{n}\right), F\right]_{d^{\prime}}
$$

be Ehresmann cycle classes of dimension $d$ and $d^{\prime}$ respectively, We say that

$$
\left[\left(a_{0}, \cdots, a_{n}\right), F\right]_{d}>\left[\left(b_{0}, \cdots, b_{n}\right), F\right]_{d^{\prime}}
$$

if for all cycles $\left(a_{0}, \cdots, a_{n}\right)$ and $\left(b_{0}, \cdots, b_{n}\right)$ in

$$
\left[\left(a_{0}, \cdots, a_{n}\right), F\right]_{d} \text { and }\left[\left(b_{0}, \cdots, b_{n}\right), F\right]_{d^{\prime}}
$$

respectively, $\left(a_{0}, \cdots, a_{n}\right)>_{\text {lex }}\left(b_{0}, \cdots, b_{n}\right)$.

Given any two subcollections $\mathcal{T}_{c}$ and $\mathcal{T}_{c^{\prime}}$ of distinguished monomials of degrees $c$ and $c^{\prime}$ respectively $\mathcal{T}_{c}>\mathcal{T}_{c^{\prime}}$ if for all distinguished monomials $\prod_{i=0}^{n-1} \gamma_{i}^{c_{i}}$ and $\prod_{i=0}^{n-1} \gamma_{i}^{c_{i}^{\prime}}$ in $\mathcal{T}_{c}$ and $\mathcal{T}_{c^{\prime}}$ respectively,

$$
\prod_{i=0}^{n-1} \gamma_{i}^{c_{i}}>_{\text {grlex }} \prod_{i=0}^{n-1} \gamma_{i}^{c_{i}^{\prime}} .
$$

Remark 5. The ordering on Ehresmann classes is characterized by dimensions while that of the subcollections of distinguished monomials is given by degrees.

Theorem 4 . Let $\left(a_{0}, \cdots, a_{n}\right)$ and $\left(b_{0}, \cdots, b_{n}\right)$ be any two cycles in the Ehresmann cycle class

$$
\left[\left(a_{0}, \cdots, a_{n}\right), F(n+1)\right]_{d}
$$

of dimension $d$, with distinguished monomials $\prod_{i=0}^{n-1} \gamma_{i}^{\chi\left(a_{i}\right)}$ and $\prod_{i=0}^{n-1} \gamma_{i}^{\chi\left(b_{i}\right)}$ respectively then

$$
\left(a_{0}, \cdots, a_{n}\right)>\left(b_{0}, \cdots, b_{n}\right)
$$

if and only if

$$
\prod_{i=0}^{n-1} \gamma_{i}^{\chi\left(b_{i}\right)}>_{\text {grlex }} \prod_{i=0}^{n-1} \gamma_{i}^{\chi\left(a_{i}\right)}
$$

\section{Proof}

Suppose that

$$
\left(a_{0}, \cdots, a_{n}\right)>\left(b_{0}, \cdots, b_{n}\right),
$$

from 2.1, $\chi\left(a_{i}\right)$ and $\chi\left(b_{i}\right)$ are given by

$$
\#\left\{a_{j}: a_{i}<a_{j}, 0 \leq i<j \leq n\right\}
$$

and

$$
\#\left\{b_{j}: b_{i}<b_{j}, 0 \leq i<j \leq n\right\}
$$

respectively, then there is $i_{0}, 0 \leq i_{0} \leq n$ in the two $n$-tuple exponents

$$
\begin{aligned}
& \left(\chi\left(a_{0}\right), \cdots, \chi\left(a_{i_{0}}\right), \cdots, \chi\left(a_{n}\right)\right) \\
& \left(\chi\left(b_{0}\right), \cdots, \chi\left(b_{i_{0}}\right), \cdots, \chi\left(b_{n}\right)\right) \in \mathbb{Z}_{\geq 0}^{n}
\end{aligned}
$$

such that $\chi\left(a_{i_{0}}\right)<\chi\left(b_{i_{0}}\right)$ and for all $k<i_{0} \quad \chi\left(a_{k}\right)$ coincides with $\chi\left(b_{k}\right)$, if they exist. Therefore, in the the vector difference

$$
\begin{aligned}
& \left(\chi\left(a_{1}\right)-\chi\left(b_{1}\right), \cdots, \chi\left(a_{i_{0}}\right)-\chi\left(b_{i_{0}}\right), \cdots, \chi\left(a_{n}\right)-\chi\left(b_{i_{0}}\right)\right) \\
& \in \mathbb{Z}^{n},
\end{aligned}
$$

the leftmost nonzero entry is negative and and hence

$$
\begin{aligned}
& \left(\chi\left(a_{1}\right), \cdots, \chi\left(a_{i_{0}}\right), \cdots, \chi\left(a_{n}\right)\right) \\
& >_{\text {grlex }}\left(\chi\left(b_{1}\right), \cdots, \chi\left(b_{i_{0}}\right), \cdots, \chi\left(b_{n}\right)\right)
\end{aligned}
$$

and the results follows easily. On the other hand suppose

$$
\prod_{i=0}^{n-1} \gamma_{i}^{\chi\left(b_{i}\right)}>_{\text {grlex }} \prod_{i=0}^{n-1} \gamma_{i}^{\chi\left(a_{i}\right)}
$$

this implies that

$$
\begin{aligned}
& \left(\chi\left(a_{1}\right), \cdots, \chi\left(a_{i_{0}}\right), \cdots, \chi\left(a_{n}\right)\right) \\
& >_{\text {grlex }}\left(\chi\left(b_{1}\right), \cdots, \chi\left(b_{i_{0}}\right), \cdots, \chi\left(b_{n}\right)\right),
\end{aligned}
$$

there is $i_{0}, \quad 0 \leq s_{0}, \leq n$ such that for all $t<i_{0}$, $\chi\left(b_{t}\right)-\chi\left(a_{t}\right)$ vanish, if $\chi\left(a_{t}\right), \chi\left(b_{t}\right)$ exist and

$$
\chi\left(b_{i_{0}}\right)-\chi\left(a_{i_{0}}\right)>0
$$

Let the set $\left\{c_{0}, \cdots, c_{n}\right\}$ be the natural descending order of the cycles

$$
\left(b_{0}, \cdots, b_{i_{0}}, \cdots, b_{n}\right) \text { and }\left(a_{0}, \cdots, a_{i_{0}}, \cdots, a_{n}\right) .
$$

Then $b_{i_{0}}-a_{i_{0}}$ is negative and

$$
b_{0}-a_{0}=\cdots=b_{i_{0}-1}-a_{i_{0}-1},
$$

Since $b_{i_{0}}, a_{i_{0}}$ are the

$$
\left(\chi\left(b_{i_{0}}\right)+1\right)^{\text {th }},\left(\chi\left(a_{i_{0}}\right)+1\right)^{\text {th }}
$$

elements of the sets

$$
\left\{c_{0}, \cdots, c_{n}\right\} /\left\{b_{0}, \cdots, b_{i_{0}-1}\right\} \text { and }\left\{c_{0}, \cdots, c_{n}\right\} /\left\{a_{0}, \cdots, a_{i_{0}-1}\right\}
$$


respectively and therefore $\left(a_{0}, \cdots, a_{n}\right)>\left(b_{0}, \cdots, b_{n}\right)$.

Corollary 2. Let

$$
\left[\left(a_{0}, \cdots, a_{n}\right), F\right]_{d} \text { and }\left[\left(b_{0}, \cdots, b_{n}\right), F\right]_{d^{\prime}}
$$

be Ehresmann cycle classes of dimension $d$ and $d^{\prime}$ respectively, and let their corresponding subcollections of their distinguished monomials be $\mathcal{T}_{c}$ and $\mathcal{T}_{c^{\prime}}$ of distinguished monomials of degrees $c$ and $c^{\prime}$ respectively,then

$$
\left[\left(a_{0}, \cdots, a_{n}\right), F\right]_{d}>\left[\left(b_{0}, \cdots, b_{n}\right), F\right]_{d^{\prime}}
$$

if and only if $\mathcal{T}_{c^{\prime}}>\mathcal{T}_{c}$.

Corollary 3. Let

$$
\begin{aligned}
& {\left[\left(a_{0}, \cdots, a_{n}\right), F\right]_{d_{0}},\left[\left(a_{0}, \cdots, a_{n}\right), F\right]_{d_{1}}, \cdots,} \\
& {\left[\left(a_{0}, \cdots, a_{n}\right), F\right]_{d_{n}}}
\end{aligned}
$$

be Ehresmann cycles classes in the flag manifold $F(n+1)$ of dimensions $d_{0}, \cdots, d_{n}$ respectively such that $d_{0}<d_{1}<\cdots<d_{n}$, and Let $\mathcal{T}_{c_{n}}, \mathcal{T}_{c_{n-1}}, \cdots, \mathcal{T}_{c_{0}}$ their corresponding subcollections of distinguished monomials of degrees $c_{n}, \cdots, c_{0}$ respectively, such that $c_{n}>\cdots>c_{0}$ then the relation

$$
\begin{aligned}
& {\left[\left(a_{0}, \cdots, a_{n}\right), F\right]_{d_{n}}>\left[\left(a_{0}, \cdots, a_{n}\right), F\right]_{d_{n-1}}} \\
& >\cdots>\left[\left(a_{0}, \cdots, a_{n}\right), F\right]_{d_{0}}
\end{aligned}
$$

induces the relation $\mathcal{T}_{c_{n}}>\mathcal{T}_{c_{n-1}}>\cdots>\mathcal{T}_{c_{0}}$ vice versa.

\section{Acknowledgements}

The first author would like to acknowledge the support provided by Education Trust Fund(Nigeria), University of Ibadan and University of New Mexico. Albuquerque, USA.

\section{REFERENCES}

[1] C. Ehresmann, "Sur la Topologie de Certains Espaces Homogene,” Annals of Mathematics, Vol. 35, No. 2, 1934, pp. 396-443. http://dx.doi.org/10.2307/1968440

[2] S. A. Ilori, "On the Geometry of Flag Manifolds and Flag Bundles. Presentata dal Corrisp. E. Martinelli,” Accademia Nazionale dei Lincei, Vol. 62, 1977, pp. 180-183.

[3] D. Monk, “The Geometry of Flag Manifolds,” Proceedings London Mathematical Society, Vol. 9, No. 2, 1959, pp. 252-286.

[4] A. Borel, "Sur La Cohomologie des Espace Fibres Principaux et des Espaces Homogenes de Groups de Lie Compacts,” Annals of Mathematics, Vol. 57, No. 1, 1953, pp. 115-207.

[5] A. Borel, "Topology of Lie Groups and Characteristic Classes,” Bulletin of the American Mathematical Society, Vol. 61, No. 5, 1953, pp. 397-432. http://dx.doi.org/10.1090/S0002-9904-1955-09936-1

[6] W. Fulton, "Flags, Schubert Polynomials, Degeneracy Loci and Determinantal Formulas," Duke Mathematical Journal, Vol. 65, No. 3, 1992, pp. 381-420.

[7] A. Grothendieck, “Anneaux de Chow et Applications Chapter IV,” Seminaire Chevalley, 1958.

[8] F. Hirzebruch, "Topological Methods in Algebraic Geometry,” Springer, Berlin, Reprint, 1978.

[9] A. Kohnert, "Weintrauben, Polynome, Tableaux," Bayreuther Mathematische Schriften, Vol. 38, 1991, pp. 1-97.

[10] F. Sottile, "Pieri’s Formula for Flag Manifolds and Schubert Polynomials," Annales de L'institut Fourier (Grenoble), Vol. 46, No. 1, 1996, pp. 89-110. 